\title{
2020 Acknowledgments
}

Psychology of Popular Media is very fortunate to have a wide pool of talented reviewers to complement the editorial board. The Editor would like to thank the following scholars for contributing their expertise and energy.

George Aalbers

Aciah Abdulsater

Aubrie Adams

Cassandra Alexopoulos

Johnie J. Allen

Phyllis Anastasio

LaNaya Anderson

Kathryn Anderson

Mara S. Aruguete

Hanan Asghar

Diane Ashe

Anita Atwell Seate

Olusola Ayandele

Nor Jijidiana Azmi

Amanda Baker

Eleftherios Baltzidis

Kim Barbour

Christopher Barry

Sheri Bauman

Anthony Martin Bean

Elizabeth Behm-Morawitz

Patrick Bender

McCall Booth

Nevfel Boz

Marianna Braoun

Britney G. Brinkman

Jeanne Brockmyer

Warren Brodsky

Ngoc Hong Bui

Melinda Burgess

Kaitlyn Burnell

Manuel Cargnino

Daniel P. Chadborn

Sarah Chan

Grace Yiseul Choi

Sophia Choukas-Bradley

Sally Chung

Annabel Cohen

Paola Corsano

Katherine Cotter

Mark Coulson

Ryan Scott Creech

Di Cui

Serena Daalmans

Leah Dajches

Katherine Dale

Kristian Daneback

Elizabeth A. Daniels

Samantha Daskaluk

William Davis

Whitney DeCamp

Lauren J. DeCarvalho

Nova Deighton-Smith
Martin Arnaud Delhove

Jaye L. Derrick

Benjamin Detenber

Maria Di Blasi

Sarah Diefenbach

Leyla Dogruel

Kristin Drogos

Royette Tavernier Dubar

Eric F. DuBow

Mark Duffett

Jennifer Dyer-Seymour

Carla Earhart

Sarah Erickson

Seda Erzi

Shir Etgar

Carlo Fabricatore

Lien Faelens

Chris Fradkin

C. Joseph Francemone

Niki Fritz

Antonis Gardikiotis

Hyungjin Gill

Jacob Glazier

Carlos Allende Gonzalez Velazquez

Gordon Goodman

John Goodwin

Donald Grant

C. Shawn Green

Keegan Greenier

Ralf Greenwald

Tobias Greitemeyer

Andrea Guazzini

Dustin Andrew Hahn

Andrew Hales

Alice Hall

Jake Harwood

Linda Heath

Veronica Hefner

Joseph Hilgard

Cynthia Hoffner

Joseph Hoffswell

Patrick Hogan

Jim Houran

Tom Hummer

Nicole E. Iannone

Juan-Jose Igartua

Ruud Jacobs

Michael Jenkins-Guarnieri

Nancy Jennings

Brian D. Johnson

Benjamin K. Johnson
Amanda Michelle JonesRincon

Kelly A. Kane

Sarah A. Kass

Linda K. Kaye

Michael Keesler

Nóra Kerekes

David Kidd

Janna Kim

Monica Koehn

Constantinos Kokkinos

Amanda Kramer

Valerie Ellen Kretz

Eugene Kukshinov

Atul Kumar Goyal

Sebastian Kurtenbach

Lauren Lane

Jens Lange

Mickey Langlais

Travis Langley

Soon Li Lee

Linda Levitt

Julia R. Lippman

Sara Magee

Hemali Maher

Anne Maheux*

Matthew C. Makel

Srikant Manchiraju

Cade Mansfield

Timothy Marjoribanks

Hayley McCullough

Lauren McInroy

Miranda McIntyre

Pilar Medina-Bravo

Elisa Mekler

Jessica S. Mendoza

Ewa Miedzobrodzka

Andreas Miles-Novelo

M. Rohangis Mohseni

Anne Montgomery

Kimberly More*

Todd Morrison

Sarah Murnen

Jessica Gall Myrick

Kimberly Neuendorf

Thuy-vy Nguyen

Rune Kristian Lundedal

Nielsen

Taylor Nocera

Oluyinka Ojedokun

Cheryl Olson

Stephanie Orme
Alanna Peebles

Robert Perl

Alison Phillips

Eva Pujadas

Jessie Quintero Johnson

Benson Rajan

Arthur Raney

Apara Ranjan

Stephen Reysen

Karyn Riddle

Rachel Rodgers

Sara Rodriguez-Cuadrado

Daniel Dan Romer

Lawrence Rubin

Nicolas Ruth

Pamela Rutledge

Roscoe Scarborough

Molly Schlesinger

Syed Siraj

Martin Slama

Ashton Speno

Alexander Spradlin

Constance Steinkuehler

Jennifer Stevens Aubrey

Chris Stiff

Kaveri Subrahmanyam

Yi-Yuan Tang

Luca Tateo

István Tóth-Király

Alvin Tran

Jolien Trekels

Jessica Troilo

Leora Trub

Riva Tukachinsky

Tia Tyree

Laura Vandenbosch

Megan A. Vendemia

Alexandra Voinescu

Jan Benjamin Vornhagen*

Abigail Walsh

L. Monique Ward

Apichai Wattanapisit

Halie Wenhold

Jeffrey Wimmer

He Xiao*

Haley Yaremych

Cleoputri Yusainy

Gila Cohen Zilka

Melanie Jo Zimmer-Gembeck

* Denotes a reviewer who co-reviewed under the supervision of a primary reviewer. 